\title{
Quantitative ED(X)S: The Zeta-Factor Method
}

\author{
Meiken Falke ${ }^{1}$, Andi Käppel ${ }^{1}$, Igor Nemeth ${ }^{1}$ and Ralf Terborg ${ }^{1}$ \\ ${ }^{1}$ Bruker Nano GmbH, Am Studio 2D, 12489 Berlin, Germany
}

Energy dispersive X-ray spectroscopy is by now well-established for qualitative and quantitative composition analysis of electron transparent samples in SEM, TEM and STEM and is reaching from the $\mathrm{mm}$ to the atomic scale. Single mobile atomic impurities in non-ideal real life samples can be identified within seconds using high-end STEM instrumentation [1-2], which is a remarkable step towards analyzing the role of single atoms in various materials science problems, e.g. impurities on nanoparticle surfaces in catalysis.

Although single atom spectroscopy is feasible now, the correct quantitative composition analysis of larger element mixtures, particularly mixtures of heavy and light elements still is a problem worth consideration. We report on the implementation of the $\zeta$-factor method [2-4] as an absolute EDS quantification method for electron transparent samples and opposed to the widely used relative CliffLorimer method. The latter can provide quantitative data on the accuracy level of a few at $\%$ and if using large solid and take-off angles even ppm already. The quantitative results from the Cliff-Lorimer method are either based on theoretical Cliff-Lorimer factors, calculated from cross-section and fluorescence yield data for particular elements at specific electron beam energies, or are only valid relative to a standard.

An alternative absolute quantification procedure, the $\zeta$-factor method, has been developed by $\mathrm{M}$. Watanabe. The method relies on having more information than in case of the relative Cliff-Lorimer approach. The electron dose must be known for all measurements and additionally, for the standard measurement, the standard thickness and density must be known. The approach then allows the quantification of sample compositions while accounting for absorption and fluorescence effects and determining e.g. the thickness for an unknown sample of interest. A further advantage is that from the $\zeta$ factors obtained with one standard measurement the $\zeta$-factors for all the other elements can be obtained from the, afore mentioned, theoretically calculated Cliff-Lorimer factors.

The $\zeta$-factor method has been implemented into the Bruker ESPRIT 2.0 software and tested using various standards such as Al- and Ti oxides, $\mathrm{GaP}$ [5] and $\mathrm{Si}_{3} \mathrm{~N}_{4}$. For an initial test procedure a $30 \mathrm{~nm}$ $\mathrm{Si}_{3} \mathrm{~N}_{4}$ foil (commercially available from Agar) was used as a standard and the same material with $60 \mathrm{~nm}$ thickness was used as a test specimen. For this the foil was damaged by the electron beam to produce sample areas with folds of known thickness and composition. The spectrum from one of these areas (Fig 1) was processed to determine the net count number for the individual $X$-ray lines and then to compute the respective $\zeta$-factors for $\mathrm{Si}-\mathrm{K}$ and $\mathrm{N}-\mathrm{K}$. Those $\zeta$-factors were then tested on a $\mathrm{Si}_{3} \mathrm{~N}_{4}$ sample region of a different well known thickness and vice versa.

The experimentally determined $\zeta$-values can be used to calculate a proportionality factor to the respective Cliff-Lorimer factors, theoretically obtainable from available atomic data for any beam energy. Based on the experimentally specified $\zeta /$ Cliff-Lorimer factor ratio the Zeta-factors for all element K-lines can then be calculated (Fig.2) and used to quantify other sample compositions using the 
same instrumentation. Our tests suggest that for the method to be successful, the beam current and the thickness and composition of the standard must be known precisely [6].

[1] T. C. Lovejoy et al., Proceedings of M\&M (2015), this volume.

[2] R. M. Stroud et al., Proceedings of M\&M (2015), this volume.

[3] M. Watanabe, Z. Horita, and M. Nemoto, Ultramicroscopy 65 (1996) 187-198.

[4] M. Watanabe \& D.B.Williams, J. of Micr. Vol. 221. (2006) 89-109.

[5] G. Kothleitner, et al., Microsc. Microanal. 20, (2014), 678-686.

[6] We gratefully acknowledge helpful discussions with W. Grogger, G. Kothleitner and S. Fladischer from the ZELMI in Graz, Austria and sample courtesy of K. Voltz, University of Marburg, Germany
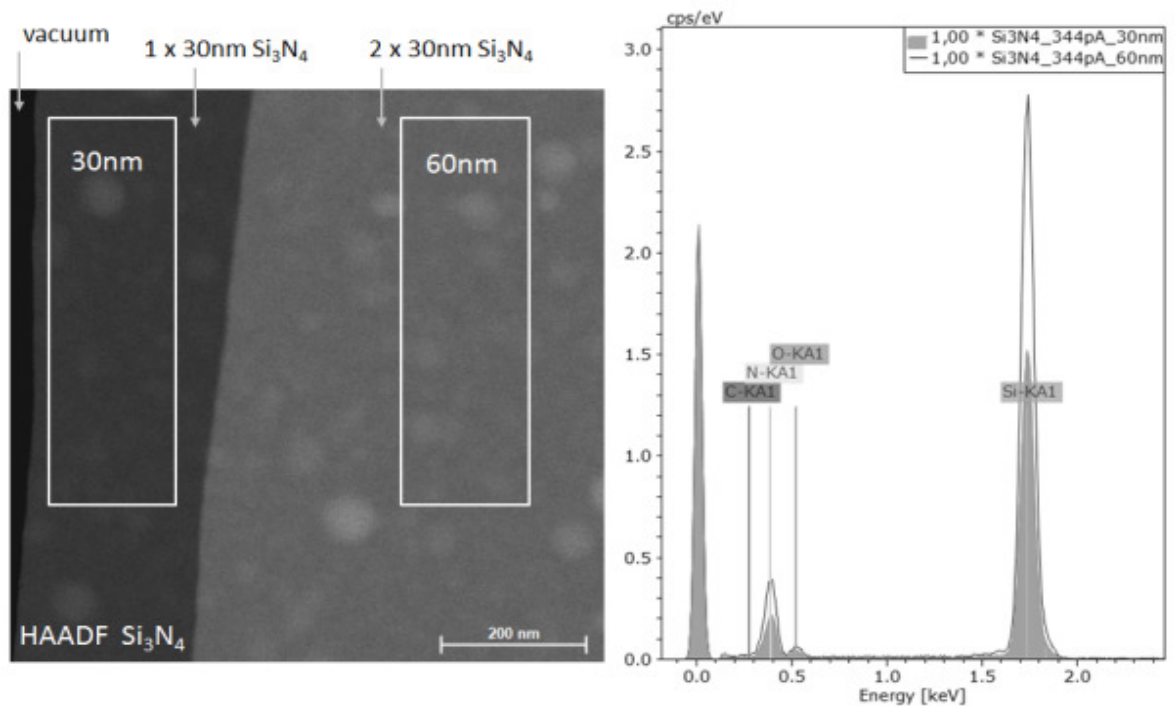

Figure 1. Two areas of the $\mathrm{Si}_{3} \mathrm{~N}_{4}$ foil and the respective spectra used for testing the Zeta-factor method.

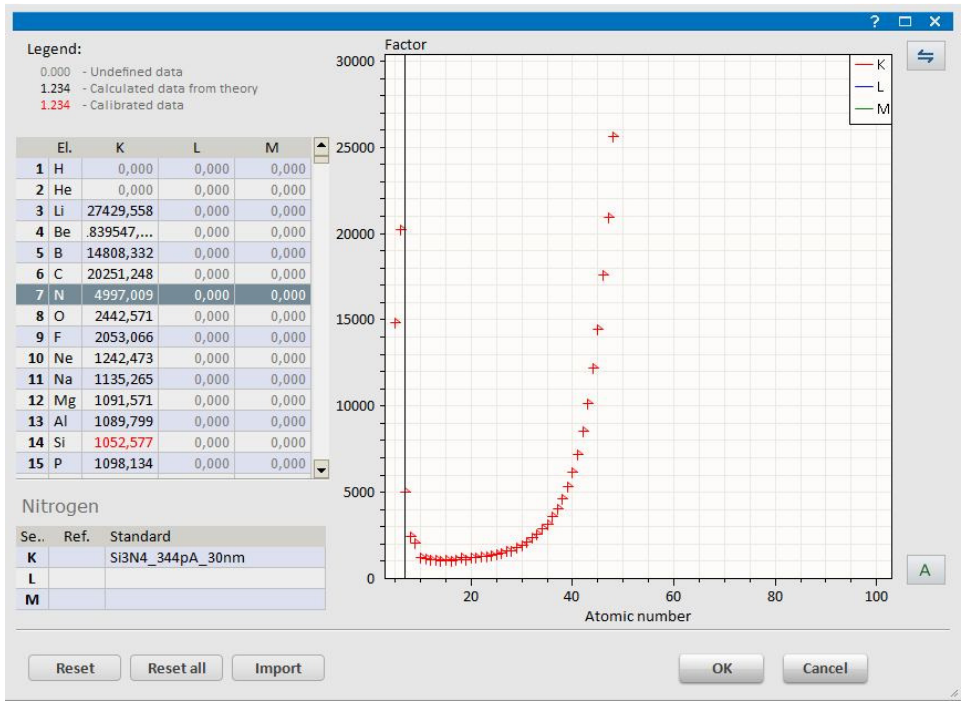

Figure 2. $\zeta$-factors for $\mathrm{K}$ lines calculated for $200 \mathrm{kV}$ based on the theoretically determined CliffLorimer-factors and the $\mathrm{N}$ - and $\mathrm{Si}$ - $\zeta$-factors obtained experimentally using $\mathrm{Si}_{3} \mathrm{~N}_{4}$ as standard. 